\title{
On the Traces of Operators (from Grothendieck to Lidskii)
}

\author{
Didier Robert (Université de Nantes, France)
}

1

\section{Presentation}

In this paper, the reader's attention is drawn to some notions that are classical in linear algebra but become more subtle to deal with in the context of infinite-dimensional vector spaces (endowed with a norm or a vector space topology). The Fredholm theory of integral equations, which will be mentioned at the end of the article, shares many common points with the systems of linear equations taught in the first year of undergraduate studies, except that the endomorphisms operate on Banach spaces of the form $C(K)$ (the space of continuous functions on a compact $K$ ) or $L^{p}(\Omega)$ for a given measure on $\Omega$. In Fredholm theory, the notions of trace and determinant, as well as their relations to eigenvalues, of course play the same role as in finite dimension. Fredholm's seminal article "Sur une classe d'équations fonctionnelles" [Acta Mathematica, 27, pp. 365-390] goes back to 1903, a time when functional analysis was poorly developed. It was the starting point of many works that partially motivated the advances in the field throughout the 20th century (Hilbert, Banach, Fréchet, Dieudonné, Schwartz, Grothendieck, Sobolev, Gelfand, Krein and many others).

More recently, microlocal analysis and the theory of pseudo-differential operators have allowed major progress in understanding the non-self-adjoint operators that describe the instability of certain systems in fluid or quantum mechanics. Indeed, the location in the complex plane of the eigenvalues with non-zero imaginary part enables one to get qualitative information about the resonance of the system (see Zworski's paper [18] for an illustration of these phenomena). The recent work [16] by Sjöstrand on Weyl formulas for randomly perturbed non-self-adjoint operators clearly shows the interest of determinants in infinite dimension. By exploiting their subtle properties as entire functions on the complex plane, one can obtain information about the eigenvalues. Lidskii's trace formula plays a pivotal role in these studies.

The aim here is to tell the history of this formula and review its role in the developments of functional analysis, as well as its revival in recent years. At the end, a sketch is presented of a proof that is close to the original one.

\section{Introduction}

On a complex vector space $\mathcal{E}$, of finite dimension $n$, the trace and the determinant of an endomorphism $A$ have the following two basic properties: they are invariant under conjugation by automorphisms and can naturally be expressed in terms of the eigenvalues of $A$ (using a basis for which $A$ is triangular).

Let us briefly recall some well known results from linear algebra. Let $\left\{e_{1}, \cdots, e_{n}\right\}$ be a basis of $\mathcal{E}$ and let $\left\{e_{1}^{*}, \cdots, e_{n}^{*}\right\}$ be the dual basis of the dual vector space $\mathcal{E}^{*}$. We denote by $\mathcal{L}(\mathcal{E})$ the $\mathbb{C}$-vector space of endomorphisms of $\mathcal{E}$.

The trace of $A \in \mathcal{L}(\mathcal{E})$ is defined by the equality

$$
\operatorname{Tr}(A)=\sum_{1 \leq j \leq n} e_{j}^{*}\left(A e_{j}\right)
$$

It is a linear form on $\mathcal{L}(\mathcal{E})$.

Let us now turn to the determinant. We write $u \wedge v$ for the exterior product of two linear forms $u$ and $v$ on $\mathcal{E}$. We denote the alternating $n$-linear form on $\mathcal{E}^{n}$ by $L_{n}=e_{1}^{*} \wedge e_{2}^{*} \cdots \wedge e_{n}^{*}$, the symmetric group on $\{1, \ldots, n\}$ by $\Im_{n}$ and the sign of $\sigma$ by $\varepsilon_{\sigma}$. Then, we have:

$$
L_{n}\left(x_{1}, \cdots, x_{n}\right)=\sum_{\sigma \in \mathbb{S}_{n}} \varepsilon_{\sigma} e_{1}^{*}\left(x_{\sigma(1)}\right) \cdots e_{n}^{*}\left(x_{\sigma(n)}\right)
$$

for all $\left(x_{1}, \cdots, x_{n}\right) \in \mathcal{E}^{n}$. The determinant of an endomorphism $A$ of $E$ is defined by the following equality:

$$
\begin{aligned}
\operatorname{det} A & =L_{n}\left(A e_{1}, \cdots, A e_{n}\right) \\
& =\sum_{\sigma \in \mathbb{S}_{n}} \varepsilon_{\sigma} e_{1}^{*}\left(A e_{\sigma(1)}\right) \cdots e_{n}^{*}\left(A e_{\sigma(n)}\right) .
\end{aligned}
$$

It is the unique complex number such that, for all alternating $n$-linear forms $f$ on $\mathcal{E}^{n}$ and all $\left(x_{1}, \cdots, x_{n}\right) \in \mathcal{E}^{n}$, one has

$$
f\left(A x_{1}, \cdots, A x_{n}\right)=(\operatorname{det} A) f\left(x_{1}, \cdots, x_{n}\right) .
$$

It follows that $\operatorname{det}(A B)=(\operatorname{det} A)(\operatorname{det} B)$ for all $A, B \in \mathcal{L}(\mathcal{E})$.

In particular, $\operatorname{det} A$ does not depend on the chosen basis. By picking a basis for which $A$ is triangular, we deduce that $\operatorname{det} A=\lambda_{1} \lambda_{2} \cdots \lambda_{n}$, where $\lambda_{j}$ are the eigenvalues of $A$. Therefore, the characteristic polynomial of $A$ can be written as

$$
D_{A}(z)=\operatorname{det}(A-z \mathbb{1})=\prod_{1 \leq j \leq n}\left(\lambda_{j}-z\right) .
$$

In the above formula, the eigenvalues are repeated according to their multiplicity.

Thanks to (2), we obtain a formula for the coefficients of the characteristic polynomial involving traces. As we shall see, it extends to infinite dimension. For this, we introduce the tensor powers $\otimes^{k} \mathcal{E}(k \geq 1)$ and the antisymmetrisation operator $\Pi_{a}$, defined for $x_{1}, \cdots, x_{k} \in \mathcal{E}$ by

$\Pi_{a}\left(x_{1} \otimes x_{2} \otimes \cdots \otimes x_{k}\right)=\frac{1}{k !} \sum_{\sigma \in \mathfrak{S}_{k}} \varepsilon_{\sigma} x_{\sigma(1)} \otimes x_{\sigma(2)} \otimes \cdots \otimes x_{\sigma(k)}$.

Given $A_{j} \in \mathcal{L}(\mathcal{E}), 1 \leq j \leq k$, we define an endomorphism of $\otimes^{k} \mathcal{E}$ by

$$
A_{1} \wedge A_{2} \cdots \wedge A_{k}=\Pi_{a}\left(A_{1} \otimes A_{2} \cdots \otimes A_{k}\right) \Pi_{a} .
$$

Setting $\wedge^{k} A=A \wedge A$ k times $\wedge A$, one has:

$$
D_{A}(-z)=z^{n}+z^{n-1} \operatorname{Tr}(A)+\cdots+z^{k} \operatorname{Tr}\left(\wedge^{k} A\right)+\cdots+z^{0} \operatorname{det} A .
$$


Notice that $\operatorname{det} A=\operatorname{Tr}\left(\wedge^{n} A\right)$. The traces in $\mathcal{L}\left(\otimes^{k} \mathcal{E}\right)$ are computed in the basis

$$
\left\{e_{j_{1}} \otimes e_{j_{2}} \otimes \cdots \otimes e_{j_{k}},\left(j_{1}, j_{2}, \cdots, j_{k}\right) \in\{1, \cdots, n\}^{k}\right\} .
$$

In particular, using the property analogous to the one we saw for the determinant, one has $\operatorname{Tr}\left(B^{-1} A B\right)=\operatorname{Tr}(A)$ for all invertible $B \in \mathcal{L}(\mathcal{E})$, and $\operatorname{Tr} A=\lambda_{1}+\cdots+\lambda_{n}$.

Finally, the trace is the unique linear form on $\mathcal{L}(\mathcal{E})$ that is invariant under conjugation up to multiplication by a constant: if $f$ is a linear form on $\mathcal{L}(\mathcal{E})$ such that $f\left(B^{-1} A B\right)=f(A)$ for all $A, B \in \mathcal{L}(\mathcal{E})$, with $B$ invertible, then there exists $\mu \in \mathbb{C}$ such that $f(A)=\mu \operatorname{Tr} A$ for all $A \in \mathcal{L}(\mathcal{E})$. The proof of this property is left to the reader as an exercice (hint: first consider rank one endomorphisms).

In finite dimension, there are, of course, several ways to obtain these results; the above presentation has the advantage of remaining valid in infinite dimension. In that case, the notions of trace and determinant are harder to deal with, even for Hilbert spaces, since there is a priori no equivalent technique to the triangulation for arbitrary operators.

To analyse the spectrum of non-diagonalisable compact operators (which appear, for instance, in the study of dissipative systems and in the Fredholm theory of integral equations), it is very useful to be able to define a trace and a determinant with reasonable properties, analogous to the ones we just recalled in finite dimension.

Let us consider a compact operator $A$ on $\mathcal{H}$. We know that, besides 0 , the spectrum of $A$ consists of a sequence of eigenvalues $\left\{\lambda_{j}(A)\right\}_{j \geq 1}$ with finite multiplicity. The multiplicity $\mu(\lambda)$ of the eigenvalue $\lambda \neq 0$ is equal to

$$
\mu(\lambda)=\operatorname{dim}\left[\mathcal{E}_{\lambda}(A)\right], \text { where } \mathcal{E}_{\lambda}(A):=\bigcup_{k \geq 1} \operatorname{ker}(A-\lambda \mathbb{1})^{k}
$$

is the generalised eigenspace. The convention used here will be to write the sequence $\left\{\lambda_{j}(A)\right\}_{j \geq 1}$ with each eigenvalue repeated according to its multiplicity.

In 1959, the Russian mathematician V. B. Lidskii proved [9] that if $\mathcal{H}$ is a separable Hilbert space and $A$ is a trace class operator (as defined below) on $\mathcal{H}$ then, for each orthonormal basis $\left\{e_{n}\right\}$ of $\mathcal{H}$, one has

$$
\sum_{n \geq 1}\left\langle e_{n}, A e_{n}\right\rangle=\sum_{j \geq 1} \lambda_{j}(A) .
$$

Here, $\langle\cdot, \cdot\rangle$ denotes the scalar product on $\mathcal{H}$, which is assumed to be anti-linear with respect to the first argument. We write $\|\cdot\|$ for the norm on $\mathcal{H}$ defined by the scalar product.

Equality (5) might look like a trifling extension of the finite-dimensional case. However, it was not until 1959 that a proof was published (although Grothendieck implicitly had all that was needed, he did not state the result explicitly). As we shall see, Lidskii's proof relies on classical, yet tricky, arguments involving subtle properties of entire functions.

To begin with, we recall a definition of the HilbertSchmidt and the trace classes (the terminology "nuclear operator" instead of "trace class operator" is also used).

Definition 2.1. An operator $A$ on $\mathcal{H}$ is in the Hilbert-Schmidt class if there is an orthonormal basis $\left\{e_{n}\right\}_{n \geq 0}$ of $\mathcal{H}$ such that

$$
\sum_{n \geq 1}\left\|A e_{n}\right\|^{2}<+\infty
$$

One shows that the left side of (6) is independent of the chosen orthonormal basis and that this condition implies that $A$ is compact. We will say that $A$ is a trace class operator if there exists a decomposition $A=A_{1} A_{2}$, where $A_{1}$ and $A_{2}$ are in the Hilbert-Schmidt class. A positive operator $A$ is in the trace class if and only if $\sum_{j \geq 1} \lambda_{j}(A)<+\infty$.

The sets of Hilbert-Schmidt and trace class operators are denoted by $\Xi_{2}(\mathcal{H})$ and $\Xi_{1}(\mathcal{H})$ respectively. We write $\Xi_{\infty}(\mathcal{H})$ for the set of compact operators and $\mathcal{L}(\mathcal{H})$ for the set of bounded operators. As the reader will have already guessed, there exist classes $\mathfrak{\subseteq}_{p}(\mathcal{H})$ for all real numbers $p>0$ : an operator $A$ belongs to $\widetilde{S}_{p}(\mathcal{H})$ if and only if $\left(A^{*} A\right)^{p / 2}$ is a trace class operator (here, $A^{*}$ denotes the Hermitian adjoint of $A$ ). For $p \geq 1$, these are Banach spaces with respect to some natural norms $\|\cdot\|_{p}$. We shall often use the convention $\|\cdot\|_{\infty}=\|\cdot\|$ (uniform norm for bounded operators). The spaces $\Xi_{p}(\mathcal{H})$ are normed two-sided ideals in the $C^{*}$-algebra $\mathcal{L}(\mathcal{H})$ (see below).

The spaces $\mathfrak{\Xi}_{p}(\mathcal{H})$ were introduced by von Neumann and Schatten and carry the name of Schatten class operators (see [4] for their properties). They share common features with the Lebesgue spaces $L^{p}$ (for a space with a measure). The functional trace "Tr" plays the role of the integral, as one has, for instance, the relation $\operatorname{Tr}\left(A^{*} A\right)=\|A\|_{2}^{2}$.

It is then clear that, if $A$ is a trace class operator, one can define its trace by the natural formula

$$
\operatorname{Tr} A=\sum_{n \geq 1}\left\langle e_{n}, A e_{n}\right\rangle .
$$

The fact that the series converges absolutely is a consequence of the Cauchy-Schwarz inequality. One can easily check that $\operatorname{Tr}$ is a continuous linear form on $\mathfrak{S}_{1}(\mathcal{H})$ that is independent of the chosen orthonormal basis. It satisfies $\operatorname{Tr} A^{*}=\overline{\operatorname{Tr} A}$ and $\operatorname{Tr}(A B)=\operatorname{Tr}(B A)$ for all $A \in \mathcal{S}_{1}(\mathcal{H})$ and $B \in \mathcal{L}(\mathcal{H})$.

It has been known since H. Weyl (see [4]) that if $A$ is a trace class operator then the series $\sum_{j} \lambda_{j}(A)$ is absolutely convergent (we shall give more details below). We can now rigorously state the result proved by Lidskii.

Theorem 2.2 (Lidskii [9]). For all trace class operators $A$, equality (5) holds.

This can be considered as the fundamental theorem in spectral analysis of non-self-adjoint operators. Indeed, the eigenvalues of an operator are, in general, difficult to access (even in the self-adjoint case). One way to control them is to write a trace formula

$$
\operatorname{Tr}(f(A))=\sum_{j \geq 1} f\left(\lambda_{j}(A)\right)
$$

in such a way that the left side can be analysed and estimated for a suitable family of functions $f$ depending on a real or a complex parameter. One then concludes by a tauberian-type argument (see for instance [1]).

Furthermore, if $A$ is assumed to be a normal operator, meaning that $A A^{*}=A^{*} A$, then Lidskii's theorem is of course trivial, since $A$ is then diagonalisable. However, in the general case, we are faced with a subtle and deep theorem. Due to the instability of the spectrum of compact non-self-adjoint operators, it is not easy to go from finite to infinite dimension.

Detailed proofs can be found in a number of books $[3,4,10,15]$. Notice that all of them except [3] explicitly 


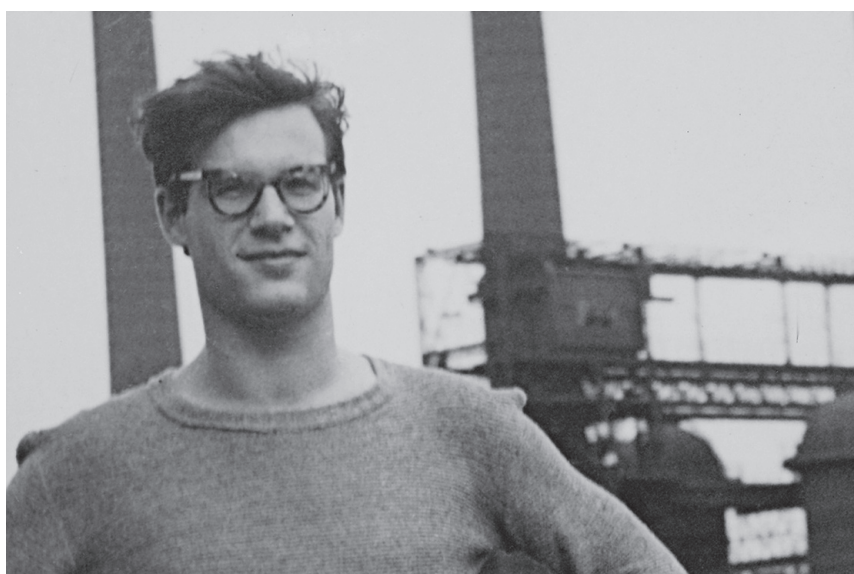

Alexander Grothendieck

attribute the result to Lidskii. His original paper (1959), written in Russian, was translated in 1965, whereas [3] appeared in 1963. Lidskii's original proof was revisited and simplified in [4]. The basic ideas will be explained at the end of this article. The proofs mentioned above use the properties of determinants as entire functions of one complex variable. Other proofs, more algebraic in nature, are based on the search for triangular forms in infinite dimension [12].

In his monumental thesis, published in [5], Grothendieck considerably developed the question of defining a trace (and a determinant) for general classes of operators on Banach or Fréchet spaces. He wrote his thèse d'État under the supervision of J. Dieudonné and L. Schwartz at Nancy University and defended it in 1953. The reader is invited to look at the four pages that L. Schwartz dedicates to A. Grothendieck in his memoir book "A mathematician grappling with his century" [pp. 282-286, Birkhäuser 2001]. Recall that Grothendieck received the Fields Medal in 1966 for his work in algebraic geometry.

One of the motivations of his doctoral research was to establish a general setting for Fredholm theory, as an extension of the Schwartz kernel theorem [6]. Nevertheless, one question seemed not to have been solved: when applied to the particular case of Hilbert spaces, Grothendieck's results definitely give a proof of (5) but only for a more restrictive class of operators than the natural trace class $\Xi_{1}(\mathcal{H})$, namely $\widetilde{\Xi}_{2 / 3}(\mathcal{H})$.

In [10, Section (27.4.11)], A. Pietsch obtained a sufficient condition for equality (5) to hold in an arbitrary Banach space. It contains the trace formulas of Grothendieck and Lidskii as particular cases. Grothendieck's approach to establish (5) for Banach spaces is also discussed in [11].

\section{Grothendieck's approach to the trace equality (5)}

Grothendieck's thesis [5] is devoted to locally convex topological vector spaces, as well as the various classes of operators between them. Here, we shall limit ourselves to Banach spaces (see also [6]).

In this section, $\mathcal{E}$ denotes a complex Banach space, $\mathcal{L}(\mathcal{E})$ denotes the algebra of bounded operators on $\mathcal{E}$ and $\mathcal{E}^{\prime}$ denotes the topological dual of $\mathcal{E}$. We write $\langle\cdot, \cdot\rangle$ for the duality (note that the bracket is bilinear) and $\mathcal{L}_{F}(\mathcal{E})$ for the ideal of finite rank operators, which we identify with the tensor product $\mathcal{E}^{\prime} \otimes$ $\mathcal{E}$ via the linear map $J\left(x^{\prime} \otimes x\right) y=\left\langle y, x^{\prime}\right\rangle y$.

We define the ideal $\mathcal{N}(\mathcal{E})$ of nuclear operators by introducing on $\mathcal{E}^{\prime} \otimes \mathcal{E}$ the so-called projective norm

$$
\|u\|_{\pi}=\inf \left\{\sum_{j \geq 1}\left\|x_{j}^{\prime}\right\|\left\|x_{j}\right\|, u=\sum_{j \geq 1} x_{j}^{\prime} \otimes x_{j}\right\}
$$

and denote by $\mathcal{E}^{\prime} \hat{\otimes}_{\pi} \mathcal{E}$ the completion with respect to this norm.

The canonical injection $J$ extends to a continuous linear map $J_{\pi}$ from $\mathcal{E}^{\prime} \hat{\otimes}_{\pi} \mathcal{E}$ to $\mathcal{L}(\mathcal{E})$. In general, $J_{\pi}$ is not injective (see [5]) but it is for Hilbert spaces. Most of the Banach spaces that one actually uses have this property (for example, the spaces $L^{p}$ for any measure $\mu$ and any $\left.1 \leq p \leq+\infty\right)$, which is related to the approximation property (see [5]). The first counterexample is due to P. Enflo [1973].

The set $\widetilde{S}_{1}(\mathcal{E})$ of nuclear operators on $\mathcal{E}$ is the image of $\mathcal{E}^{\prime} \hat{\otimes}_{\pi} \mathcal{E}$ in $\mathcal{L}(\mathcal{E})$ by $J_{\pi}$. It is a two-sided ideal and a Banach space with respect to the quotient norm on $\mathcal{E}^{\prime} \hat{\otimes}_{\pi} \mathcal{E} / \operatorname{ker} J_{\pi}$.

In what follows, we always assume that $J_{\pi}$ is injective.

For any $A$ of finite rank, the trace is naturally defined as $\operatorname{Tr} A=\sum x_{j}^{\prime}\left(x_{j}\right)$ if $A=J(u)$ and $u=\sum_{j}\left\langle x_{j} \otimes x_{j}^{\prime}\right\rangle$. One can easily show that it extends by continuity to $\Xi_{1}(\mathcal{E})$, yielding a linear form such that $|\operatorname{Tr}(A)| \leq\|A\|_{1}$ for all $A \in \widetilde{\Xi}_{1}(\mathcal{E})$. Moreover, the trace is invariant on $\Im_{1}(\mathcal{E})$, in that $\operatorname{Tr}(T A)=$ $\operatorname{Tr}(A T)$ for all $T \in \mathcal{S}_{1}(\mathcal{E})$ and all $A \in \mathcal{L}(\mathcal{E})$.

We introduce, on the Banach space $\mathcal{E}$, a family $\mathfrak{\Xi}_{p}(\mathcal{E})$ of ideals for all real numbers $p>0$. For this, let $A$ be a continuous linear operator on $\mathcal{E}$.

Definition 3.1. We say that the operator $A$ is $p$-summable if there exist sequences $x_{j}$ in $\mathcal{E}$ and $x_{j}^{\prime}$ in $\mathcal{E}^{\prime}$ with $\left\|x_{j}\right\|=\left\|x_{j}^{\prime}\right\|=1$, and a sequence of positive real numbers $\sigma=\left\{\sigma_{j}\right\}$ such that $\sum_{j \geq 1} \sigma_{j}^{p}<+\infty$ and

$$
A(u)=\sum_{j \geq 1} \sigma_{j}\left\langle u, x_{j}^{\prime}\right\rangle x_{j}, \forall u \in \mathcal{E} .
$$

We denote by $\widetilde{\Xi}_{p}(\mathcal{E})$ the ideal of $p$-summable operators. Clearly, one has $\Im_{p}(\mathcal{E}) \subseteq \Im_{q}(\mathcal{E})$ if $p \leq q$. The 1-summable operators are exactly the trace class (or nuclear) operators. The trace is then given by

$$
\operatorname{Tr}(A)=\sum_{j \geq 1} \sigma_{j}\left\langle x_{j}, x^{\prime}\right\rangle .
$$

For this definition to make sense, the right side of (10) must be independent of the representation (9) of $A$. This is the case whenever $\mathcal{E}$ has the approximation property, since $J_{\pi}$ is then injective.

Clearly, every $p$-summable operator for $p>0$ is compact. Let $\lambda_{j}$ be the non-zero eigenvalues of $A$ represented as many times as their algebraic multiplicities. In [5] (Ch. I, pp. 171177 and Ch. II, p. 20), Grothendieck obtained the following result.

Theorem 3.2 (Grothendieck). Suppose that $A$ is $2 / 3$ summable. Then, the following equality holds:

$$
\operatorname{Tr}(A)=\sum_{j \geq 1} \lambda_{j}(A)
$$

Theorem 3.2 is optimal for general Banach spaces: for example, in the Banach space $\ell_{1}$ of summable sequences, there 
exists an operator $N$ that is $p$-summable for all $p>2 / 3$ and such that $N^{2}=0$ and $\operatorname{Tr}(N)=1$ ([10], paragraph 10.4.5).

It might come as a surprise that Grothendieck did not tackle the particular case of Hilbert spaces in a more explicit way. In Chapter II of his thesis (p. 13), he claims: "If $p \leq 1$ then the Fredholm determinant of $u$ has genus 0." In our notation, $u=A$ is assumed to be $p$-summable. The next section recalls the properties of Fredholm determinants, as well as the definition of the genus of an entire function (see below, following formula (17)).

At the end of [9], Lidskii adds that he learned about Grothendieck's work [5] (including the above quotation) when his article was in press. Although the combination of the genus zero property, the results on Fredholm theory [6] and the factorisation theorem of entire functions by WeierstrassHadamard [7] give a proof of Theorem 2.2, Grothendieck neither stated the result nor pursued the argument to the end. This observation was noted in several publications, in particular in [11].

Theorems 2.2 and 3.2 were unified by Pietsch [10] as follows. We denote by $\ell_{p}$ the space of sequences of complex numbers whose $p$-th powers are summable and we denote by $p^{\prime}$ the real conjugate of $p$. In [10], the author studies many families of ideals of operators. In particular, he introduces the following ideals in the Banach space $\mathcal{B}$.

Definition 3.3. Let $r, p, q$ be three real numbers such that $r>$ 0 and $1+\frac{1}{r} \geq \frac{1}{p}+\frac{1}{q}$. The operator $A \in \mathcal{L}(\mathcal{B})$ is called $(r, p, q)$ nuclear if there exists a factorisation $A=S D_{\text {iag }}(\sigma) R$ such that $R \in \mathcal{L}\left(\mathcal{B}, \ell_{q^{\prime}}\right), S \in \mathcal{L}\left(\ell_{p}, \mathcal{B}\right)$ and $\sigma \in \ell_{r}$, where $D_{\text {iag }}(\sigma)$ denotes the diagonal operator associated with $\sigma$.

We write $\mathfrak{A}_{(r, p, q)}(\mathcal{B})$ for the set of $(r, p, q)$ nuclear operators of $\mathcal{B}$. Note that $A$ belongs to $\mathfrak{N}_{(r, p, q)}(\mathcal{B})$ if and only if $A$ admits a representation

$$
A=\sum_{j \geq 1} \sigma_{j} x_{j}^{\prime} \otimes x_{j},
$$

where $\sigma \in \ell_{r},\left(x_{j}\right) \in \ell_{p^{\prime}}(\mathcal{B})$ and $\left(x_{j}^{\prime}\right) \in \ell_{p^{\prime}}\left(\mathcal{B}^{\prime}\right)$. Here, $\ell_{p}(\mathcal{B})$ denotes the space of sequences of $\mathcal{B}$ that are weakly in $\ell_{p}$. In particular, $\mathfrak{N}_{(r, 1,1)}$ agrees with the set of $r$-summable operators on $\mathcal{B}$ and, given a Hilbert space $\mathcal{B}=\mathcal{H}$, one has $\mathfrak{N}_{(1,1,2)}(\mathcal{H})=\mathfrak{S}_{\infty}(\mathcal{H})$.

Theorem 3.4 (Pietsch [10]). Let $A \in \mathfrak{N}_{(1,1,2)}(\mathcal{B})$ be an operator. Then, $\sum_{j}\left|\lambda_{j}(A)\right|<+\infty$ and

$$
\operatorname{Tr} A=\sum_{j \geq 1} \lambda_{j}(A) .
$$

It is easy to see that $\mathfrak{R}_{(2 / 3,1,1)}(\mathcal{B}) \subseteq \mathfrak{N}_{(1,1,2)}(\mathcal{B})$. This theorem contains the trace equalities of Grothendieck and Lidskii.

Remark 3.5. Note that, in his proof, Pietsch does not use the approximation property for $\mathcal{B}$.

In 1988, Pisier [11] introduced a class of "weak Hilbert" Banach spaces that are characterised by a condition on the weak type and cotype. He shows that, in these spaces, one has $\operatorname{Tr} A=\sum_{j \geq 1} \lambda_{j}(A)$ for any nuclear operator $A$ satisfying $\sum_{j \geq 1}\left|\lambda_{j}(A)\right|<+\infty$.

\section{Traces and invariant functions}

One can easily show that any invariant continuous linear form $f$ on $\Xi_{1}(\mathcal{B})$ is a multiple of the trace $\operatorname{Tr}$ (see the introduction). It is natural to look for other invariant functions on $\mathfrak{S}_{1}(\mathcal{B})$, particularly for polynomial functions on other ideals of $\mathcal{L}(\mathcal{B})$.

Let us mention that Dixmier studied another property of the trace: normality. Let $\mathcal{L}_{+}(\mathcal{H})$ be the cone of positive operators on an infinite-dimensional separable Hilbert space $\mathcal{H}$. We call a trace any function $f: \mathcal{L}_{+}(\mathcal{H}) \rightarrow[0,+\infty]$ that is positive, additive and homogeneous. We say that $f$ is a normal trace if it is, moreover, completely additive: if $A=\sum_{n \geq 1} A_{n}$, with $A_{n} \in \mathcal{L}_{+}(\mathcal{H})$, then $f(A)=\sum_{n \geq 1} f\left(A_{n}\right)$. It is easy to see that any normal trace is proportional to the usual trace $\mathrm{Tr}$.

Dixmier proved that $\mathcal{L}_{+}(\mathcal{H})$ possesses a non-normal trace $\operatorname{Tr}_{\mathrm{D}}$, nowadays called a Dixmier trace. This trace is identically zero for finite rank operators. In his book "Non-commutative geometry" [Academic Press, Inc., 1994], A. Connes reproduces the article by Dixmier and gives an application to perturbative field theory.

We are now going to consider the invariant polynomial functions that appear naturally in the Fredholm theory of determinants.

Definition 4.1. A two-sided ideal $\mathfrak{S}$ of $\mathcal{L}(\mathcal{E})$ is said to be normed if it is equipped with a norm $\|\cdot\|_{\odot}$ such that

$$
\|R A S\|_{\Im} \leq\|R\|\|A\| \subseteq\|S\| .
$$

A continuous function $f$ with complex values on the ideal $\subseteq$ is invariant if $f\left(T^{-1} A T\right)=f(A)$ for all $A \in \subseteq$ and all $T \in \mathcal{L}(\mathcal{E})$. This property amounts to $f(A T)=f(T A)$ for all $T \in \mathcal{L}(\mathcal{E})$.

The spaces $\mathfrak{\Xi}_{1}(\mathcal{E}), \mathfrak{\Xi}_{\infty}(\mathcal{E})$ and $\mathfrak{S}_{p}(\mathcal{H})$, for $1 \leq p<+\infty$, are all normed ideals.

In this section, we shall determine all polynomial functions that are invariant under a normed ideal $\mathfrak{S}_{1}(\mathcal{E})$, as well as under the Schatten classes $\Im_{p}(\mathcal{H})$ in the Hilbert case. This computation was carried out independently in [13] and [2] for different purposes: in [13], it was to justify a numerical method to find the eigenvalues of systems of elliptic partial differential equations, initiated by Fichera in the work "Linear elliptic systems and eigenvalue problems" [Lecture Notes in Math. No. 8, Springer-Verlag-1965); and in [2], it was to study classifying spaces of vector bundles.

We start by computing the invariant polynomial functions on the ideal $\mathcal{L}_{F}(\mathcal{E})$. Recall that a homogeneous polynomial function of degree $n$ on a Banach space $\mathcal{B}$ is a map $\Phi$ from $\mathcal{B}$ to $\mathbb{C}$ defined by a continuous $n$-linear symmetric form $\tilde{\Phi}$ such that $\Phi(A)=\tilde{\Phi}\left(A,{ }^{n}\right.$ times,$\left.A\right)(\tilde{\Phi}$ is unique $)$. Following [6], we obtain the fundamental invariant forms by a tensor computation.

We denote by $\otimes^{n} \mathcal{E}$ the $n$-th tensor power of $\mathcal{E}$ and we use the natural identification between $\otimes^{n}\left(\mathcal{E}^{\prime}\right)$ and $\left(\otimes^{n} \mathcal{E}\right)^{\prime}$. Let $\Lambda_{n}$ (resp. $\Lambda^{n}$ ) be the antisymmetrisation operator on $\otimes^{n} \mathcal{E}$ (resp. $\left.\otimes^{n}\left(\mathcal{E}^{\prime}\right)\right)$. Then, $\Lambda_{n}$ is a projector on $\otimes^{n} \mathcal{E}$ and $\Lambda_{n}^{\prime}=\Lambda^{n}$. Given $A_{j} \in \mathcal{L}(\mathcal{E})$, for $j=1, \cdots, n$, one defines

$$
A_{1} \wedge A_{2} \wedge \cdots \wedge A_{n}=\Lambda_{n}\left(A_{1} \otimes A_{2} \cdots \otimes A_{n}\right) \Lambda_{n} .
$$

The assignment $\left(A_{1}, \cdots, A_{n}\right) \mapsto A_{1} \wedge A_{2} \wedge \cdots \wedge A_{n}$ is $n$-linear and symmetric. Moreover, $\wedge^{n} \mathcal{L}_{F}(\mathcal{E}) \subseteq \mathcal{L}_{F}\left(\otimes^{n} \mathcal{E}\right)$. 
One can check that, for any two integers $s, n \geq 1$, the function

$$
A \mapsto \operatorname{Tr}\left(\wedge^{s}\left(A^{n}\right)\right):=\mathcal{J}_{s}^{n}(A)
$$

is an invariant polynomial function of degree $s n$ on $\mathcal{L}_{F}(\mathcal{E})$. In fact, $\mathcal{J}_{s}^{n}$ is an elementary invariant of type $(s, n)$. Setting $\mathcal{J}_{0}^{n}=1$, the following recurrence relation holds:

$$
\mathcal{J}_{s}^{n}(A)=\frac{1}{s} \sum_{q=1}^{q=s} \mathcal{J}_{1}^{n q}(A) \mathcal{J}_{s-q}^{n}(A) .
$$

Using an inequality for determinants due to Hadamard [3, (p. 1018)], as well as the Stirling formula, one obtains:

$$
\left|\mathcal{J}_{s}^{n}(A)\right| \leq \gamma_{s}\|A\|_{1}^{n s} .
$$

In the above, $\gamma_{s} \leq C\left(e^{2} / s\right)^{\frac{s+1}{2}}$ for a universal constant $C$ in the general case and $\gamma_{s}=1 / s$ ! for Hilbert spaces. It follows that $\mathcal{J}_{s}^{n}(A)$ extends by continuity to an invariant function on $\Im_{1}(\mathcal{E})$, which satisfies, in particular, (12) and (13).

Theorem 4.2 ([2,13]). Assume that $\mathcal{E}$ has infinite dimension. The vector space $\mathcal{P}_{n}$ of homogeneous polynomial functions of degree $n \geq 1$ that are invariant under $\mathcal{S}_{1}(\mathcal{E})$ has finite dimension $p(n)$, equal to the number of partitions of $n$ as a sum of positive integers. Moreover, each of the following two families is a basis for $\mathcal{P}_{n}$ :

$$
\begin{aligned}
& \left\{\left(\mathcal{J}_{1}^{1}\right)^{r_{1}}\left(\mathcal{J}_{1}^{2}\right)^{r_{2}} \cdots\left(\mathcal{J}_{1}^{n}\right)^{r_{n}},\right\}_{r_{1}+2 r_{2}+\cdots+n r_{n}=n}, \\
& \left\{\left(\mathcal{J}_{1}^{1}\right)^{r_{1}}\left(\mathcal{J}_{2}^{1}\right)^{r_{2}} \cdots\left(\mathcal{J}_{n}^{1}\right)^{r_{n}},\right\}_{r_{1}+2 r_{2}+\cdots+n r_{n}=n} .
\end{aligned}
$$

In the case of Hilbert spaces $(\mathcal{E}=\mathcal{H})$, there is a similar statement for the Schatten classes $\Xi_{p}(\mathcal{H}), 1 \leq p<+\infty$ ([13]). In particular, any invariant polynomial function of degree $<p$ is identically zero.

The elementary invariants can be expressed in an arbitrary orthonormal basis $\left\{e_{k}\right\}$ of $\mathcal{H}$. For this, it is convenient to introduce the Hilbert tensor product defined as follows: if $\mathcal{H}_{1}$ and $\mathcal{H}_{2}$ are two Hilbert spaces, the sesquilinear form given by $\left\langle x_{1} \otimes x_{2}, y_{1} \otimes y_{2}\right\rangle=\left\langle x_{1}, y_{1}\right\rangle\left\langle x_{2}, y_{2}\right\rangle$ defines a scalar product on $\mathcal{H}_{1} \otimes \mathcal{H}_{2}$. The Hilbert tensor product is the Hilbert space $\mathcal{H}_{1} \hat{\otimes}_{2} \mathcal{H}_{2}$ obtained as the completion of $\mathcal{H}_{1} \otimes \mathcal{H}_{2}$.

Let $A$ be such that $A^{n}$ is in the trace class. Then, $\wedge^{s} A^{n}$ is in the trace class in the Hilbert space $\hat{\otimes}_{2}^{s} \mathcal{H}$ and one has

$$
\mathcal{J}_{s}^{n}(A)=\operatorname{Tr}\left(\wedge^{s} A^{n}\right) .
$$

From this, we derive

$$
\mathcal{J}_{s}^{n}(A)=\frac{1}{s !} \sum_{k_{1}, \cdots, k_{s}} \operatorname{det}_{1 \leq i, j \leq s}\left\langle e_{k_{j}}, A^{n} e_{k_{i}}\right\rangle .
$$

Theorem 2.2 yields an expression of $\mathcal{J}_{s}^{n}(A)$ in terms of the eigenvalues of $A$, namely, $\mathcal{J}_{s}^{n}(A)=\mathcal{T}_{s}^{n}(A)$ if

$$
\mathcal{T}_{s}^{n}(A)=\sum_{j_{1}<j_{2}<\cdots<j_{s}} \lambda_{j_{1}}(A)^{n} \lambda_{j_{2}}(A)^{n} \cdots \lambda_{j_{s}}(A)^{n} .
$$

From these properties, there are two possible ways to introduce a determinant. Since $A$ is a trace class operator, following Grothendieck's presentation [6], one can consider the Fredholm determinant

$$
\operatorname{det}(11-z A):=\sum_{k \geq 0}(-1)^{k} z^{k} \mathcal{J}_{k}^{1}(A)
$$

It is an entire function of $z \in \mathbb{C}$ of order 1 (in a Hilbert space). This follows from inequality (13).
For the other definition, one begins directly with the eigenvalues and the infinite product

$$
D_{A}(z):=\prod_{j \geq 1}\left(1-z \lambda_{j}\right) .
$$

We know (19) that $\sum_{j \geq 1}\left|\lambda_{j}\right| \leq\|A\|_{1}$, hence the infinite product defines an entire function of order 1 . Theorem 2.2 amounts to showing that $\operatorname{det}(\mathbb{1}-z A)=D_{A}(z)$ for all $z \in \mathbb{C}$.

To go further, it is useful to recall the Weierstrass factorisation theorem for entire functions (see W. Rudin, Real and Complex Analysis). The Weierstrass factors are the entire functions $E_{0}(z)=(1-z)$ and $E_{p}(z)=(1-z) \exp \left(z+\frac{z^{2}}{2}+\cdots+\frac{z^{p}}{p}\right)$ for $p \geq 1$. Let $f$ be an entire function. We denote by $m$ the multiplicity of 0 if $f(0)=0$ and by $\left\{z_{n}\right\}_{n \geq 1}$ the sequence of non-zero complex numbers such that $f\left(z_{n}\right)=0$, ordered by increasing modulus and repeated according to their multiplicity. The Weierstrass factorisation theorem says that $f$ admits a (non-unique) factorisation of the form

$$
f(z)=z^{m} \mathrm{e}^{g(z)} \prod_{n=1}^{\infty} E_{p_{n}}\left(\frac{z}{z_{n}}\right),
$$

with $g$ an entire function and $\left\{p_{n}\right\}$ a sequence of integers.

We say that $f$ has genus $\leq \mu$ if there exists a Weierstrass decomposition such that $p_{n} \leq \mu$ for all $n$ and $g$ is a polynomial of degree $\leq \mu$. The genus is the smallest positive integer that has this property. Therefore, being an entire function of genus 0 means that $f$ trivially factors over its zeros:

$$
f(z)=a z^{m} \prod_{n=1}^{\infty}\left(1-\frac{z}{z_{n}}\right),
$$

where $a$ is a constant. For more information on entire functions and their zeros, the reader is referred to, for example, B. Levin "Distribution of zeros of entire functions" [AMS Transl-1964].

In [6, Théorème 3], Grothendieck shows that the zeros of the entire function $F_{A}(z)=\operatorname{det}(\mathbb{1}-z A)$ are exactly the eigenvalues $\lambda_{j}(A)$ (counted with multiplicity). By the Weierstrass factorisation theorem, the equality $D_{A}=F_{A}$ holds, provided we can show that $F_{A}$ has genus 0 . This is how Grothendieck proceeds for general Banach spaces. Indeed, he proves [5, pp. 13-19] that, for all 2/3-summable operators $A$, the function $F_{A}$ has genus 0 . Theorem 3.2 follows from this.

Let $p \geq 2$ be an integer. Given an operator $A \in \widetilde{\Xi}_{p}(\mathcal{H})$, we introduce the regularised determinant ([3])

$$
\begin{aligned}
\operatorname{det}_{p}(\mathbb{1}-z A) & =\prod_{j}\left(1-z \lambda_{j}\right) R_{p}\left(z \lambda_{j}\right), \\
R_{p}(z) & =\exp \left[z+\frac{z^{2}}{2}+\cdots+\frac{z^{p-1}}{p-1}\right] .
\end{aligned}
$$

The function $\operatorname{det}_{p}(\mathbb{1}-z A)$ is an entire function of the variable $z \in \mathbb{C}$ whose zeros are the inverses of the non-zero eigenvalues of $A$. Therefore, if $A$ lies in $\mathfrak{\Xi}_{p}(\mathcal{H})$ with $p \geq 1$, there exists $r>0$ such that the equality

$$
\operatorname{det}_{p}(\mathbb{1}-z A)=\exp \left(\sum_{k \in \mathbb{N}} \frac{\mathcal{T}_{1}^{p+k}(A)}{p+k} z^{p+k}\right)
$$

holds for $|z|<r$. This formula goes back to Poincaré.

From Theorem 2.2, we derive the relations

$$
\mathcal{J}_{s}^{p}(A)=\sum_{1 \leq k \leq s} \frac{(-1)^{k+s}}{k !}\left(\sum_{r_{1}+r_{2}+\cdots+r_{k}=s} \frac{\mathcal{J}_{1}^{p r_{1}}(A) \cdots \mathcal{J}_{1}^{p r_{k}}(A)}{r_{1} \cdots r_{k}}\right) \text {. }
$$




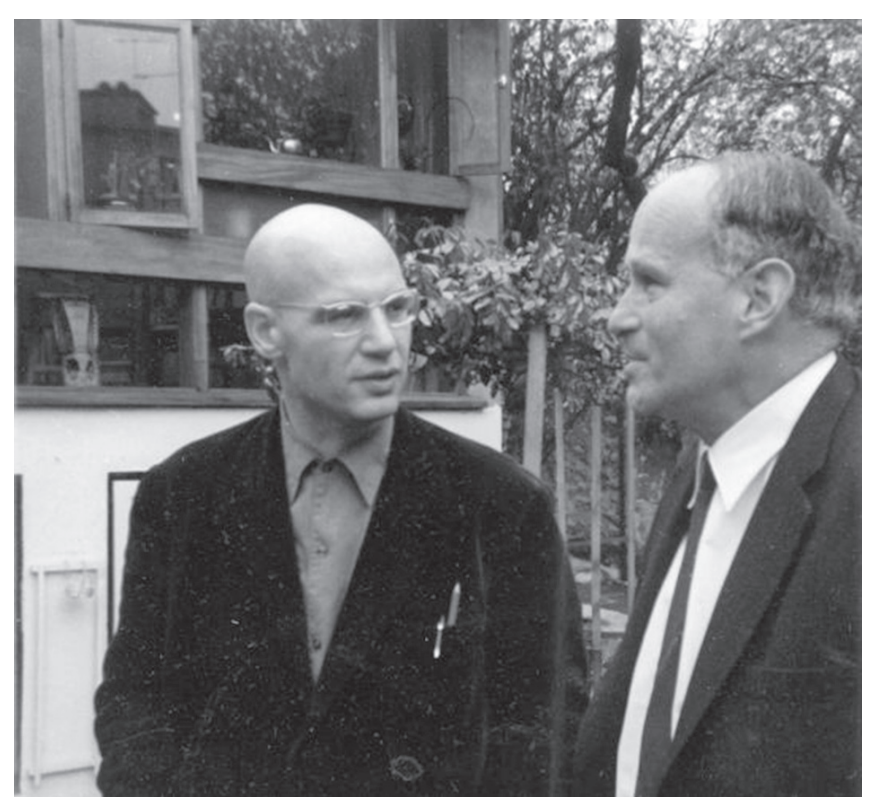

Alexander Grothendieck and Laurent Schwartz

\section{Examples}

Let us now consider the case $\mathcal{H}=L^{2}(\Omega, \mu)$, where $\mu$ is a Borel measure on a locally compact space $\Omega$. An operator $A$ on $\mathcal{H}$ is in the Hilbert-Schmidt class if and only if there exists an integral kernel $K \in L^{2}\left(\Omega \times \Omega, \mu^{\otimes 2}\right)$ such that, for $u \in L^{2}(\Omega, \mu)$, one has $A u(x)=\int_{\Omega} K_{A}(x, y) u(y) d y$. Then,

$$
\|A\|_{2}^{2}=\operatorname{Tr}\left(A^{*} A\right)=\int_{\Omega \times \Omega}\left|K_{A}(x, y)\right|^{2} d \mu(x) d \mu(y) .
$$

Let $A$ be a trace class operator of the form $A=A_{1} A_{2}$, where $A_{1}$ and $A_{2}$ are in the Hilbert-Schmidt class. It follows that $A$ has an integral kernel

$$
K_{A}(x, y)=\int_{\Omega} K_{A_{1}}(x, z) K_{A_{2}}(z, y) d \mu(z)
$$

To simplify, we suppose that $K_{A}$ is continuous on $\Omega \times \Omega$. Using Fubini's theorem, we obtain

$$
\operatorname{Tr}(A)=\int_{\Omega} K_{A}(x, x) d \mu(x),
$$

as well as the following formula for each $s \geq 1$ :

$$
\mathcal{J}_{s}^{1}(A)=\frac{1}{s !} \int_{\Omega^{s}}\left(\operatorname{det}_{\substack{1 \leq i \leq s \\ 1 \leq j \leq s}} K_{A}\left(x_{i}, x_{j}\right)\right) d \mu^{\otimes s}\left(x_{1}, \cdots, x_{s}\right) .
$$

These are classical expressions in the Fredholm theory of integral equations, which one can find, for instance, in Goursat's "Cours d'analyse mathématique" [Vol III, Gauthiers-Villars1943].

Pseudo-differential operators form an important class of examples of integral operators. On a Riemannian compact manifold $M$ of dimension $d$, a pseudo-differential operator $A$ [14] admits an integral kernel $K_{A}$ that can be written locally as

$$
K_{A}(x, y)=(2 \pi)^{-d} \int_{\mathbb{R}^{d}} a(x, \xi) \mathrm{e}^{i(x-y) \cdot \xi} d \xi
$$

where $a$ is a smooth function with complex values (it is called the symbol of $A$ ). We assume that $a$ is a classical elliptic symbol of order $m$. The principal symbol $a_{m}$ is then a homogeneous function on the cotangent space $T^{*}(M)$ that has degree $m$ in $\xi$. If $m \leq 0$ then $A$ is bounded on $\mathcal{H}:=L^{2}(M)$.
If $m<-d / p$ then $A$ is in the Schatten class $\mathfrak{\subseteq}_{p}(\mathcal{H})$. For example, in the case of the Laplace-Beltrami operator $\triangle_{M}$ on $M$, the operator $\left(-\triangle_{M}+1\right)^{-s}$ belongs to $\Xi_{p}$ if $s>\frac{d}{2 p}$. If $m<-d$ then the trace of $A$ is given (locally) by the formula

$$
\operatorname{Tr} A=(2 \pi)^{-d} \int_{T^{*}(M)} a(x, \xi) d x d \xi .
$$

Observe that, in general, the symbol $a$ is not globally defined on a manifold: only the principal symbol (the homogeneous part of highest degree) is well defined.

Symbolic calculus on pseudo-differential operators allows one to obtain information about the eigenvalues of elliptic differential operators $A$ (not necessarily self-adjoint) on a compact variety. The work of Seeley [14] is at the origin of many developments of this topic. The paper [1] contains an example of how to use these techniques to obtain asymptotic formulas for the spectrum of non-self-adjoint elliptic operators.

In the self-adjoint case, one can go much further in the study of trace formulas. For instance, the spectrum of the Laplacian on $M$ is related to geometry (Selberg, Gutzwiller); a vast literature is devoted to this subject. There are also extensions of the notion of trace to operators that are not in the trace class, for example, the relative traces (and determinants) introduced by Krein [4] and analytic continuation of a generalised zeta function [14]. As an illustration, when $A=-\triangle_{M}+1$, the function $\zeta_{A}(s):=\operatorname{Tr} A^{-s}$ is holomorphic on the complex half-plane $\{s, \mathfrak{R} s>d / 2\}$ and extends to a meromorphic function on $\mathbb{C}$ whose poles belong to the sequence $s_{j}=(d-j) / 2$. Moreover, $\zeta_{A}$ is regular at the integers [14].

\section{Sketch of the proof of Lidskii's theorem}

We first present a basic tool in the study of the eigenvalues of non-self-adjoint operators: Weyl inequalities ([4, pp. 35-41]).

Let $A$ be a compact operator on $\mathcal{H}$. We consider the sequence $\left\{\lambda_{j}(A)\right\}$ of non-zero eigenvalues (if any) ordered by increasing modulus and repeated according to their multiplicities. Let $s_{j}(A)$ be the sequence of eigenvalues of $|A|:=\sqrt{A^{*} A}$ greater than zero, the so-called singular (or characteristic) values of $A$. For any integer $N \geq 1$ and any real numbers $p, r>0$, the following inequalities hold:

$$
\begin{aligned}
\left|\lambda_{1}(A) \lambda_{2}(A) \cdots \lambda_{N}(A)\right| & \leq s_{1}(A) s_{2}(A) \cdots s_{N}(A), \\
\sum_{1 \leq j \leq N}\left|\lambda_{j}(A)\right|^{p} & \leq \sum_{1 \leq j \leq N}\left(s_{j}(A)\right)^{p}, \\
\prod_{1 \leq j \leq N}\left(1+r\left|\lambda_{j}(A)\right|\right) & \leq \prod_{1 \leq j \leq N}\left(1+r s_{j}(A)\right) .
\end{aligned}
$$

Moreover, $\|A\|_{1}=\sum_{j \geq 1} s_{j}(A)$.

Recall that $D_{A}(z):=\prod_{j, \geq 1}\left(1-z \lambda_{j}\right)$. By the third Weyl inequality, one has

$$
\left|D_{A}(z)\right| \leq \mathrm{e}^{|z||| A \|_{1}}
$$

for all $z \in \mathbb{C}$. We first assume that $A$ is in the trace class and does not have any non-zero eigenvalue. This is equivalent to $\lim _{n \rightarrow+\infty}\left\|A^{n}\right\|^{1 / n}=0$ (i.e. $A$ is quasi-nilpotent).

An elementary example of a quasi-nilpotent operator is the integration operator $K u(x)=\int_{0}^{x} u(y) d y$, which is defined on $\mathcal{H}=L^{2}([0,1])$ for the Lebesgue measure. One can immediately check that $K$ does not have eigenvalues (it is injective). It is not in the trace class but it is in the $(1+\varepsilon)$-Schatten class 
for all $\epsilon>0$. In particular, $K^{2}$ is a trace-class quasi-nilpotent operator.

Let $\Pi_{N}$ be an increasing sequence of orthogonal projectors of rank $N$ in $\mathcal{H}$ that converges strongly to the identity. Then, $A_{N}:=\Pi_{N} A \Pi_{N}$ converges to $A$ in $\widetilde{\Xi}_{1}(\mathcal{H})$ and hence in $\mathcal{L}(\mathcal{H})$. We set $\lambda_{j}^{(N)}=\lambda_{j}\left(A_{N}\right)$ and $D_{N}=D_{A_{N}}$. It follows that

$$
\lim _{N \rightarrow+\infty}\left|\lambda_{1}^{(N)}\right|=0
$$

On the other hand, computing the logarithmic derivative of $D_{N}^{\prime}(z) / D_{N}(z)$, one can show that

$$
D_{N}(z)=\exp \left(-\sum_{k \in \mathbb{N}} \frac{\mathcal{T}_{1}^{1+k}\left(A_{N}\right)}{1+k} z^{1+k}\right) .
$$

Recall that, since $A_{N}$ has finite rank $N$, the following equality holds for any integer $s$ :

$$
\mathcal{T}_{1}^{s}\left(A_{N}\right)=\operatorname{Tr}\left(A_{N}^{s}\right)=\sum_{j=1}^{N}\left(\lambda_{j}^{(N)}\right)^{s} .
$$

But the second Weyl inequality yields

$$
\left|\mathcal{T}_{1}^{1+k}\left(A_{N}\right)\right| \leq\left\|A_{N}\right\|_{1}\left|\lambda_{1}^{(N)}\right|^{k}
$$

from which it follows, writing $a=\operatorname{Tr}(A)$ and using the continuity of the trace, that

$$
\lim _{N \rightarrow+\infty} D_{N}(z)=\mathrm{e}^{-a z} .
$$

One can now prove that $\operatorname{Tr} A=0$ by arguing that if $a \neq 0$ then $D_{N}$ has polynomial growth, uniform with respect to $N$, which contradicts (21). Indeed, from the Weyl inequalities and $s_{j}\left(A_{N}\right) \leq s_{j}(A)$, one can obtain

$$
\begin{aligned}
\left|D_{N}(z)\right| & \leq \prod_{j \geq 1}\left(1+s_{j}(A)|z|\right) \\
& \leq \prod_{1 \leq j \leq M}\left(1+s_{j}(A)|z|\right) \exp \left(|z| \sum_{j \geq M+1} s_{j}(A)\right) .
\end{aligned}
$$

Choosing $M$ such that $\sum_{j \geq M+1} s_{j}(A) \leq|a| / 2$ and $z=\mathrm{e}^{-i a \arg a} r$, with $r>0$, we derive the inequality

$$
\mathrm{e}^{r|a| / 2} \leq \prod_{1 \leq j \leq M}\left(1+s_{j}(A) r\right)
$$

and hence the contradiction.

Lidskii's theorem is thus proved for quasi-nilpotent operators in the trace class. For the general case, one can decompose the Hilbert space into two orthogonal subspaces

$$
\mathcal{H}=\mathcal{H}_{D} \bigoplus \mathcal{H}_{N}, \quad \mathcal{H}_{D}=\bigoplus_{j \geq 1} \mathcal{E}_{\lambda_{j}}(A)
$$

(sum of the generalised eigenspaces for all non-zero eigenvalues). Let $P$ denote the orthogonal projector onto $\mathcal{H}_{D}$ and $P^{\perp}=\mathbb{1}-P$. Then,

$$
A=P A P+P A P^{\perp}+P^{\perp} A P+P^{\perp} A P^{\perp} .
$$

Note that $\operatorname{Tr}\left(P^{\perp} A P\right)=\operatorname{Tr}\left(P A P^{\perp}\right)=0$ (invariance of the trace) and $P^{\perp} A P^{\perp}$ is quasi-nilpotent. Indeed, $P$ commutes with $A$ and hence $A^{*}$ commutes with $P^{\perp}$. Since $A^{*}$ is quasi-nilpotent, the same holds for $P^{\perp} A P^{\perp}=\left(P^{\perp} A^{*} P^{\perp}\right)^{*}$. Finally, $P A P$ is a sum of Jordan blocks containing exactly the eigenvalues of $A$ repeated according to their multiplicities. By the linearity of the trace, this concludes the proof of Theorem 2.2.

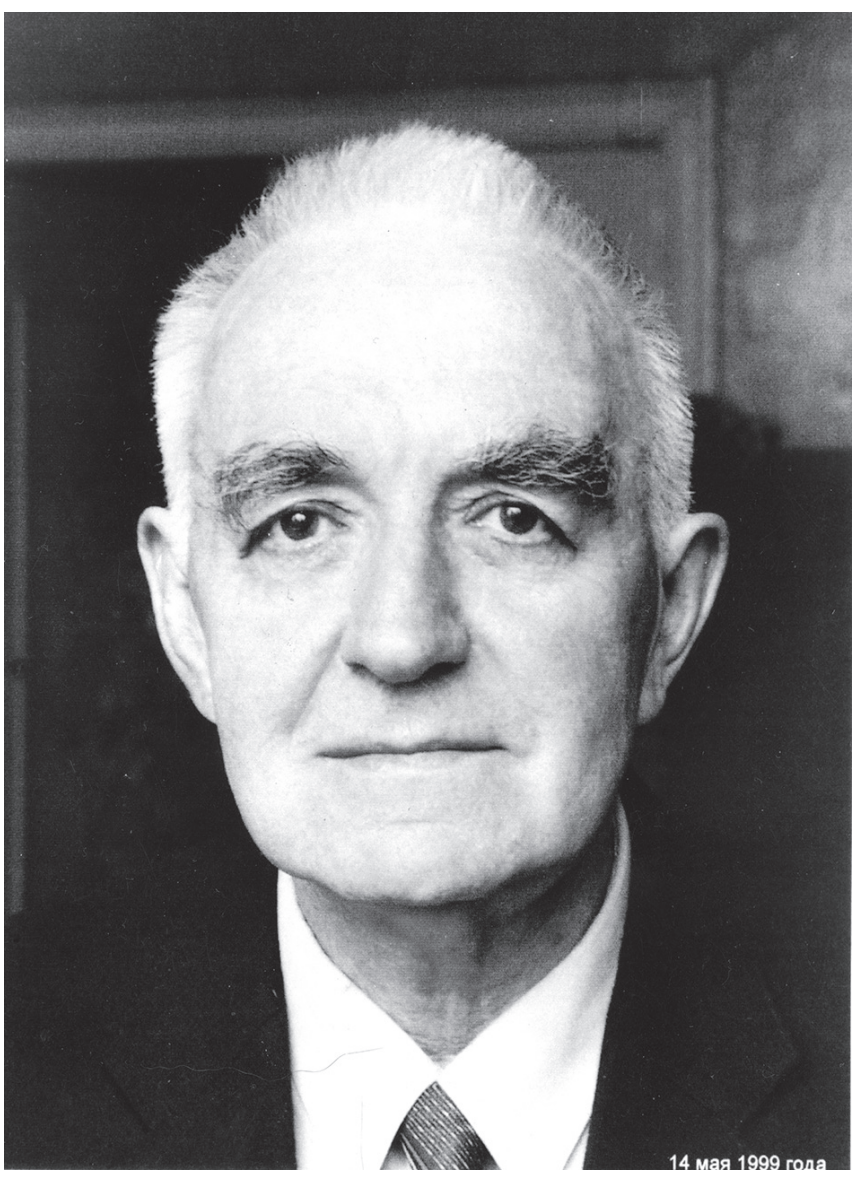

Victor Borisovich Lidskii, May 14, 1999

\section{$6 \quad$ Who was Lidskii?}

We close with a few biographical notes, referring the interested reader to the introduction of Operator Theory and its Applications [AMS Transl-2010] for more details. Edited by two of his former students, M. Levitin and D. Vassiliev, this book is devoted to Lidskii's mathematical work.

Victor Borisovich Lidskii was born in 1924 in Odessa and died in Moscow in 2008. He defended his PhD thesis "Conditions for the completeness of the system of root subspaces of non-self-adjoint operators with discrete spectra" in Moscow University in 1954, under the supervision of I. M. Gelfand. He was a professor at Moscow's Fiz Tech University from 1961 to 2008, as well as at the Institute for Problems of Mechanics of the USSR Academy of Sciences. Fiz Tech University was created in 1946 in the suburbs of Moscow to encourage research in physics (connected to the nuclear and space programmes of the USSR); it had a favoured status.

Together with Gohberg and Krein, Lidskii was one of the pioneers of spectral analysis of non-self-adjoint operators. He became a recognised expert in the field, both for theoretical aspects and applications, especially in mechanics in areas such as elasticity and hydroelasticity equations and thinshell theory. He accomplished important work in these subjects. His best known result is certainly Theorem 2.2. A renewed interest in his works arose from the recent developments on the spectrum of non-self-adjoint operators and the pseudo-spectrum (see [17] for an overview).

Another celebrated result by Lidskii deals with the extension of Weyl inequalities to eigenvalues of Hermitian matri- 
ces. Let $A$ and $B$ be two $n$ by $n$ Hermitian matrices. We denote by $\left\{\lambda_{j}(A), 1 \leq j \leq n\right\}$ the sequence of eigenvalues of $A$ in increasing order.

Theorem 6.1 (Lidskii's inequalities, 1950). For each subset $J \subseteq\{1,2, \cdots, n\}$ of cardinal $k$, one has

$$
\sum_{j \in J} \lambda_{j}(A+B) \leq \sum_{j \in J} \lambda_{j}(A)+\sum_{j=1}^{k} \lambda_{j}(B) .
$$

This important result was obtained after work by Berezin and Gelfand on Lie groups [8]. Inequality (22) is related to geometric properties concerning Schubert varieties and representation theory. A pedagogical introduction may be found in the paper by R. Bhatia, "Linear algebra to quantum cohomology: the story of Horn's inequality" [Amer. Math. Monthly2001]. Inequality (22) also has applications to numerical analysis. But this would take us too far ...

\section{Acknowledgments}

This work was supported by the ANR project "NOSEVOL" (ANR-2011-BS01019 01).

\section{References}

[1] M.S. Agranovich and A.S. Markus. On spectral proterties of elliptic pseudo differential operators far from selfadjoint ones. Zeitschrift für analysis und ihre Anwendungen, 8(3):237-260, 1989.

[2] P. de la Harpe and R. Ramer. Polynômes invariants sur les algèbres de Lie banachiques complexes classiques d'opérateurs compacts dans l'espace hilbertien. C.R.A.S Paris, pp. 824827, 1972.

[3] N. Dunford and J. T. Schwartz. Linear operators, volume II. InterScience Publishers, 1963.

[4] I. C. Gohberg and M. G. Krein. Introduction to the theory of linear nonselfadjoint operators in Hilbert space. Translations of mathematical monographs, vol. 18. A.M.S., 1969.

[5] A. Grothendieck. Produits tensoriels topologiques. A.M.S, 1955. résumé : séminaire N. Bourbaki, 1951-1954, exp. No. 69, pp. 193-200.

[6] A. Grothendieck. Théorie de Fredholm. Bull. Soc. Math. Fr., 84:319-384, 1956.

[7] B. N. Ja. Levin. Distribution of zeros of entire functions, volume 5. Amer. Math. Soc. Transl., 1964.

[8] V. B. Lidskii. On the characteristic numbers of sum and product of symmetric matrices. Dokl. Akad. Nauk. SSR, 75:769772,1950 . in russian.
[9] V. B. Lidskii. Non-selfadjoint operators with a trace. Dokl. Akad. Nauk. SSR, 125:485-487, 1959. Amer. Math. Soc. Transl. (2) 47 (1965) 43-46.

[10] A. Pietsch. Ideals of operators. North-Holland Publishing Company, 1980.

[11] G. Pisier. Weak Hilbert spaces. Proc. London Math.Soc., 56 (3):547-579, 1988.

[12] S. C. Power. Another proof of Lidskii's theorem on the trace. Bull. London Math. Soc., 15:146-148, 1983.

[13] D. Robert. Invariants orthogonaux pour certaines classes d'opérateurs compacts. C.R.A.S Paris, 273:301-304, 1971.

[14] R. Seeley. Complex powers of an elliptic operator. In Singular integrals (proc. Symp. Pure Math. 10), pages 288-307. Providence R.I.:Amer. Math. Soc., 1967.

[15] B. Simon. Trace ideals and their applications. A.M.S., 1979.

[16] J. Sjöstrand. Eigenvalue distribution for non-self-adjoint operators with small multiplicative random perturbations. Annales Fac. Sci. Toulouse, 18:4:739-795, 2009.

[17] J. Sjöstrand. Spectral properties of non-self-adjoint operators, 2009. Journées ÉDP, Évian, http://jedp.cedram.org/item?id= JEDP_2009_A1_0.

[18] M. Zworski. Resonances in Physics and Geometry. Notices of the AMS, 46:3:319-328, 1999.

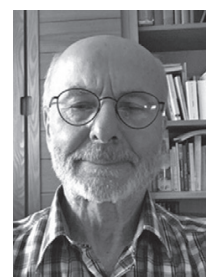

Didier Robert [didier.robert@univ-nantes. fr] has been an emeritus professor at the University of Nantes since his retirement in 2010. Before this, he was a professor in Nantes from 1981 to 2010. He started working in functional analysis and operator theory during his PhD at Poitiers University and then he moved onto partial differential equations and semiclassical and spectral analysis, with applications in quantum mechanics. He is the author of two books in these fields (Birkhauser 1987 and Springer 2012, in collaboration with M. Combescure). He is an editor of the journal Asymptotic Analysis.

This is the English translation by Javier Fresán (Centre de Mathématiques Laurent Schwartz, École Polytechnique) with corrections and modifications by the author. The French version of the text appeared in Gazette des mathématicienes (SMF), N 141 (2014), 76-94.

The EMS Newsletter is thankful to the Gazette des mathématicienes for authorisation to republish the updated version of this text. 